\title{
Aspiration einer Stiftkrone - Ursache, Therapie und Komplikationsvermeidung
}

\author{
K. Welcker \\ M. Nakashima \\ D. Branscheid
}

\begin{abstract}
Aspiration of Dental Implant - Reasons, Management and Prevention
\end{abstract}
\section{Zusammenfassung}

Die Fremdkörperaspiration bei Erwachsenen ist ein seltenes Ereignis, sie stellt allerdings eine mögliche ernstzunehmende Komplikation zahnärztlicher Behandlung dar. Wir berichten über einen 77-jährigen Patienten mit chronischem Reizhusten nach unerkannter Aspiration einer Stiftkrone. Das Implantat verblieb drei Jahre als endobronchialer Fremdkörper im Unterlappenbronchus. Eine bronchoskopische Bergung misslang, so dass bei chronischer Retentionspneumonie der rechte Unterlappen im Sinne einer Lobektomie entfernt werden musste. Eine Übersicht über Ursachen, Therapie und Komplikationsvermeidung erfolgt.

\section{Abstract:}

Aspiration of foreign bodies is extremely rare in adults, but it may occur during the course of dental treatment and become a serious problem for the patient. A case of a 77-year old man with chronic cough after dental treatment and unrecognised aspiration of an implant is presented. The implant remained unidentified for three years as an endobronchial foreign body of the lower lobe bronchus. A bronchoscopic removal failed and surgery was indicated for lobectomy of the right lower lobe because of chronic pneumonia. A review of prevention, signs and symptoms, management and documentation of the complications is presented.

\section{Einleitung}

Die Aspiration von Zahnersatz oder zahnärztlichem Kleinmaterial stellt ein seltenes Ereignis dar. Allerdings können unerkannte Fremdkörper zu nicht unerheblichen Komplikationen führen, so dass bei chronischem Husten auch differenzialdiagnostisch eine Fremdkörperaspiration in Erwägung gezogen werden muss.

\section{Fallbericht}

Wir berichten über einen 77-jährigen Patienten, der vom Hausarzt mit der Verdachtsdiagnose einer Stiftkronenaspiration in unsere Klinik eingewiesen wurde.

\begin{abstract}
Anamnese
Der Patient klagte über sechsmonatigen trockenen Reizhusten mit gelegentlichem weißlichem Auswurf. Die körperliche Untersuchung ergab rechts basal ein frühinspiratorisches Rasselgeräusch. Unter der Verdachtsdiagnose einer akuten Bronchitis erfolgte die ambulante Therapie mit N-Acetylcystein und Amoxycillin p.o. Nach einer zweiwöchigen Therapie ohne Besserung wurde eine Röntgenaufnahme des Thorax in 2 Ebenen angefertigt (Abb. 1 u. 2). In Projektion auf den rechten Unterlappenbronchus zeigte sich ein metallischer Fremdkörper mit radiologischen Zeichen einer Retentionspneumonie. Erst nach gezielter Nachfrage gab der Patient an, vor 3 Jahre zuvor bei dem Versuch der Implantation einer Stiftkrone diese angeblich „verschluckt“ zu haben. Der behandelnde Zahnarzt habe ihn lediglich gebeten
\end{abstract}

Institutsangaben

Abteilung für Thoraxchirurgie, Krankenhaus Großhansdorf, Zentrum für

Pneumologie und Thoraxchirurgie, Großhansdorf

Korrespondenzadresse

Dr. med. Katrin Welcker · Krankenhaus Großhansdorf, Zentrum für Pneumologie und Thoraxchirurgie .

Wöhrendamm 80·22927 Großhansdorf·E-mail: drwelcker@t-online.de

Eingang: 30. November 2004 · Nach Revision alkzeptiert: 23. Januar 2005

Bibliografie

Pneumologie 2005; 59: 174-177 (c) Georg Thieme Verlag KG Stuttgart · New York

DOI $10.1055 / \mathrm{s}-2004-830210$

ISSN 0934-8387 


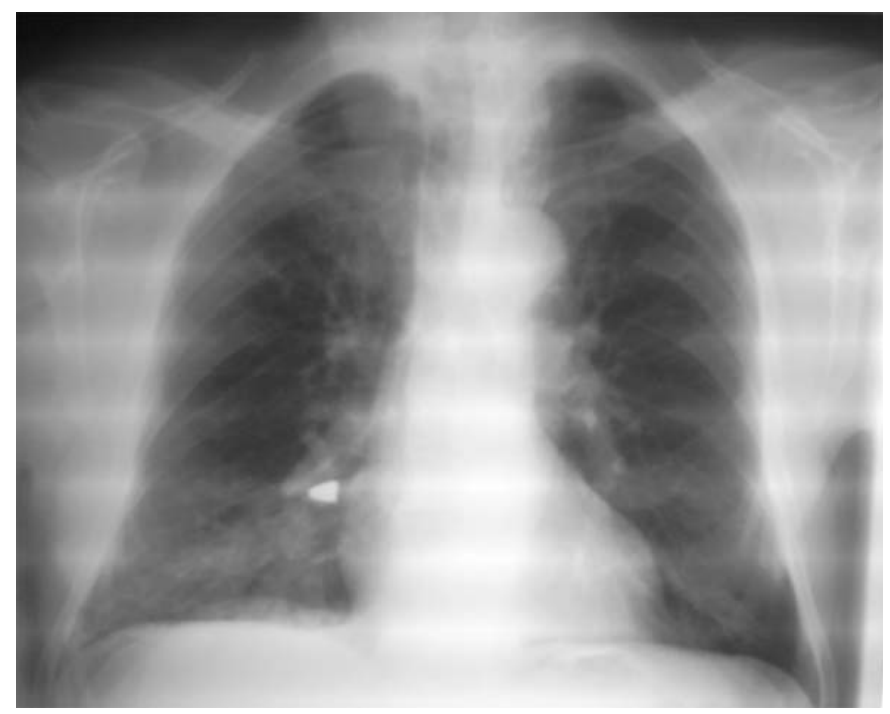

Abb. 1 Röntgenübersicht Thorax pa. Metallischer Fremdkörper im Unterlappenbronchus rechts mit begleitender Verschattung des Unterfeldes rechts.

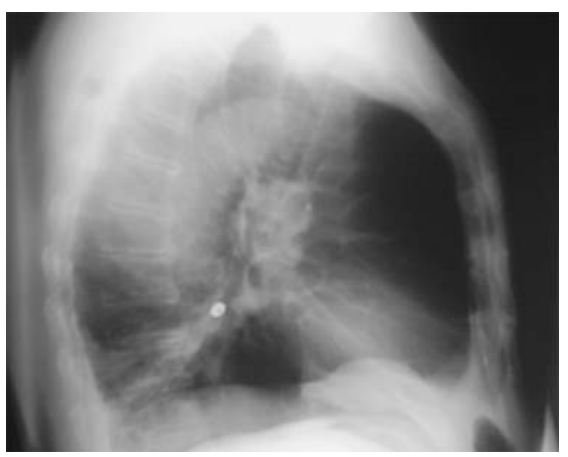

Abb. 2 Röntgenübersicht des Thorax seitlich.

„in den nächsten Tagen“ auf die Ausscheidung des Zahns mit dem Stuhlgang zu achten. Das Implantat fand sich jedoch nicht im Stuhlgang. Nachdem der Patient keine Beschwerden, insbesondere keine abdominellen Schmerzen und keinen Hustenreiz oder Dyspnoe angab, wurden von Seiten des Zahnarztes keine weiteren Maßnahmen veranlasst. Der Hausarzt wies den Patienten nun nach Anfertigung der Röntgenaufnahme unverzüglich in die pneumologisch-thoraxchirurgische Fachklinik ein.

\section{Aufnahmebefunde}

Bei stationärer Aufnahme zeigte sich ein 77-jähriger Mann, Nichtraucher $(168 \mathrm{~cm}, 72 \mathrm{~kg}$ ), in gutem Allgemeinzustand. Auskultatorisch fanden sich rechts basal grobblasige Rasselgeräusche bei ansonsten unauffälliger körperlicher Untersuchung. Die Lungenfunktion war altersentsprechend unauffällig. Zunächst wurde versucht, durch starre Bronchoskopie in Vollnarkose die aspirierte Krone endoskopisch zu bergen. Dies gelang nicht, da der Stift der Krone die Bronchuswand penetriert hatte und dort von einem derben chronischen Granulationsgewebe umgeben fest verankert war. Beim bronchoskopischen Bergungsversuch entleerte sich reichlich eitriges Sekret aus dem durch das Implantat zu zweidrittel verschlossenen Unterlappenbronchus. Somit wurde die Indikation zur operativen Bergung über eine anterolaterale Thorakotomie gestellt.

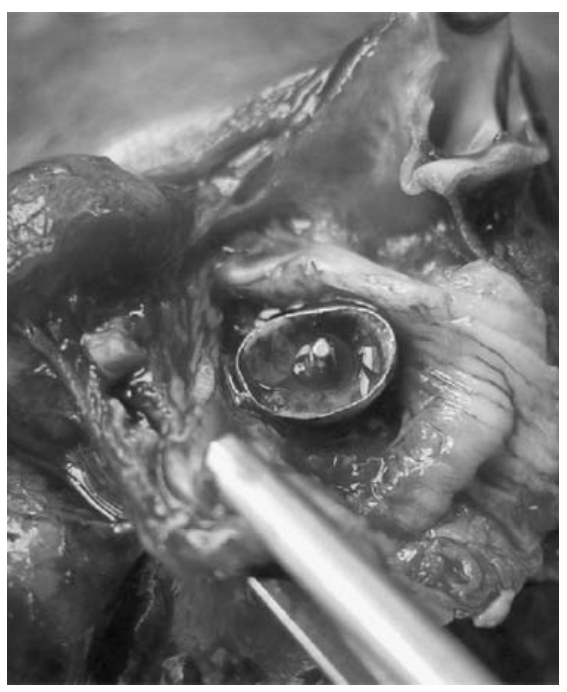

Abb. 3 Operationspräparat. Implantat im abgesetzten Unterlappenbronchus liegend.

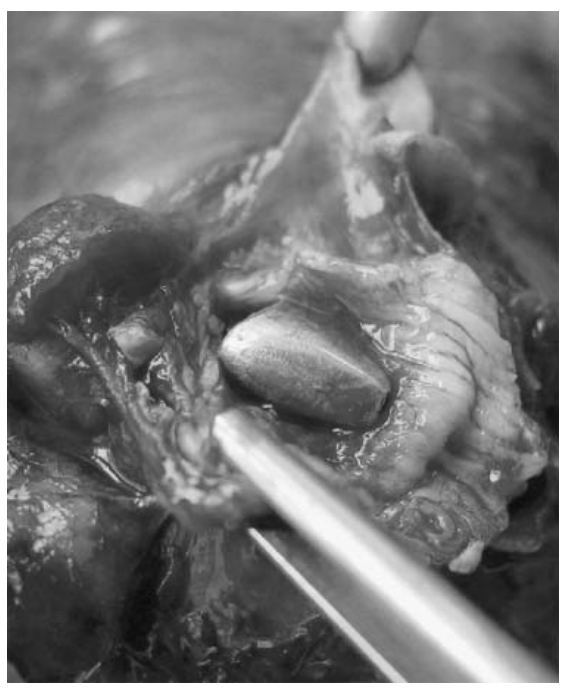

Abb. 4 Operationspräparat. Stiftkrone nach der Eröffnung des Unterlappenbronchus.

\section{Thorakotomie}

Intraoperativ zeigte sich das Implantat im Unterlappenbronchus fest verankert und mit derbem Granulationsgewebe umgeben (Abb. 3 u. 4). Der Stift des Implantates hatte die Bronchuswand penetriert. Nach dreijähriger chronischer Aspiration war der gesamte Unterlappen im Sinne einer chronischen Pneumonie ödematös derb verändert, so dass eine komplette Unterlappenresektion durchgeführt werden musste. Histologisch zeigte das Lungenpräparat Zeichen einer ausgeprägten floriden herdförmigen chronischen Pneumonie, einer eitrigen Bronchitis und Bronchiolitis sowie vereinzelten hämorrhagischen Infarkten (Abb. 5).

Der Patient erholte sich trotz seines fortgeschrittenen Alters rasch von der Operation und konnte nach einer dreitägigen Überwachung auf der Intensivstation am 16. postoperativen Tag nach Hause entlassen werden.

\section{Diskussion}

Die Fremdkörperaspiration von Zahnersatz oder Kleininstrumenten stellt eine seltene, aber ernst zu nehmende Komplikation zahnärztlicher Behandlung dar $[1,2,4,8]$. Als prädisponie- 


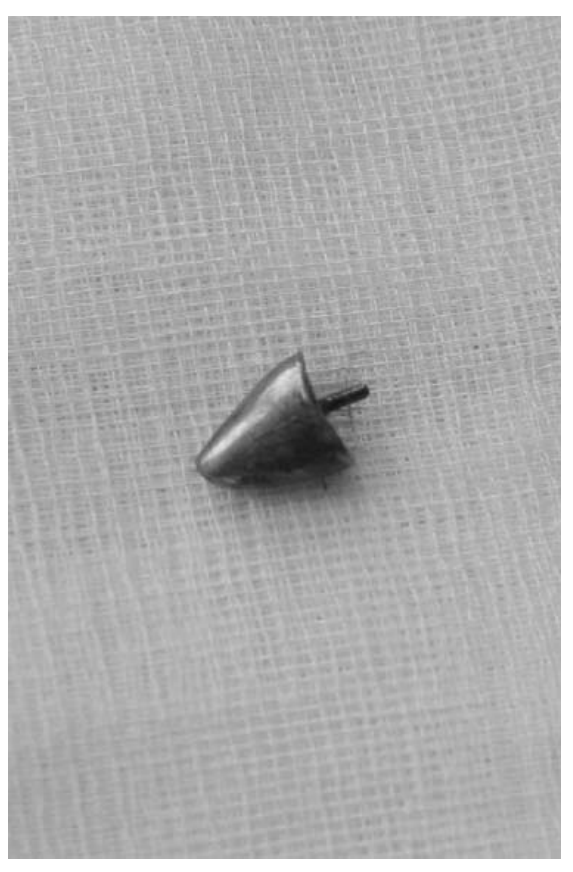

Abb. 5 Extrahiertes Implantat.

render Faktor für eine Aspiration gilt insbesondere die flache Lagerung des Patienten während der Behandlung.

Klinisch präsentiert sich die Aspiration am häufigsten mit Husten, Dyspnoe oder asthmatischen Beschwerden. Gelegentlich kommt es auch zu symptomlosen Aspirationen, so dass das Fehlen von Symptomen eine Aspiration nicht ausschließen kann [9].

Die Mehrzahl (75\%) der aspirierten Fremdkörper findet sich im steilen und weiteren rechten Hauptbronchus bzw. rechten Lungenunterlappenbronchus [4,5]. Durch Stenosierung der distalen Lungenareale und durch infizierte Wurzelkanalinstrumente oder keimbesiedelte kariöse Zähne können Infektionen bzw. eine poststenotische Pneumonie ausgelöst werden. Spitze Fremdkörper - wie in unserem Fall der Stift des Implantates -können die Bronchuswand traumatisieren bzw. perforieren und Hämoptysen oder Hämoptoe verursachen. Im Falle einer Verkeilung der Fremdkörper im Bereich der Glottis, Subglottis oder Hypopharynx kann es durch massiven Husten oder Würgen zu einer reflektorischen Vagusreizung mit Blutdruckabfall und Schocksymptomatik kommen $[10,11,13]$. Dementsprechend sollte der Patient sofort mit erhöhtem Oberkörper in eine Klinik mit der Möglichkeit der bronchoskopischen Entfernung des Fremdkörpers gebracht werden.

Diagnostisch wegweisend ist in den meisten Fällen die Röntgenaufnahme des Thorax in 2 Ebenen. Im vorliegenden Fall hätte das Implantat als metalldichter Fremdkörper sofort in der Thoraxaufnahme lokalisiert werden können. Selbst bei nichtröntgendichten Fremdkörpern finden sich in der Übersichtsaufnahme des Thorax häufig indirekte Zeichen einer Aspiration wie Atelektasen oder „air-trapping“ [3,6,12,15].

Generell gilt, jeder aspirierte Fremdkörper sollte frühestmöglich entfernt werden. Eine absolute bronchoskopische Notfallindikation stellt eine akute Fremdkörperaspiration mit Hustenanfall, plötzlicher Luftnot bis hin zur Asphyxie dar. Hier sollte bei akuter vitaler Bedrohung (Stridor, Zyanose oder Ruhedyspnoe) die unmittelbare Notfallintubation und Bronchoskopie mit dem starren Rohr erfolgen. Das Fremdkörpermaterial kann dann mit der Fasszange oder anderen geeigneten Instrumenten geborgen werden.

Im Falle einer chronischen Aspiration führt der häufig sessile Fremdkörper zu einer Schleimhautreaktion mit Bildung von Granulationsgewebe und sekundärer entzündlicher Stenose die durchaus tumorös imponieren kann.

In solchen Fällen sollte nach Inspektion im Rahmen einer flexiblen Bronchoskopie durch anschließende starre Bronchoskopie eine vorsichtige Präparation und Extraktion unter Vermeidung von stärkeren Blutungen oder einer Bronchuswandperforationen versucht werden.

Bei chronisch granulomatös ummauerten Aspiraten wie in diesem Fall gelingt in etwa 5\% der Fälle wegen Einwachsen und Penetration die endoskopische Mobilisierung nicht. Eine Thorakotomie ist dann unvermeidlich, oftmals verbunden mit Lungenparenchymresektionen bzw. einer Lobektomie. Dagegen gelingt die Fremdkörperextraktion nach akuter oder subakuter Aspiration fast immer [5,9].

In unserem Fall hätte der Zahnarzt, auch wenn er davon ausging, dass bei fehlender Atemnot und fehlendem Hustenreiz der Zahn verschluckt worden sei, unverzüglich eine Röntgenübersicht des Thorax bzw. des Abdomens zur genauen Lokalisation des Zahnersatzes veranlassen müssen. Zwar ist das Verschlucken von stumpfen Fremdkörpern für den Patienten meist bedeutungslos, da diese nach reichhaltiger ballastreicher Kost via naturales abgehen, doch können spitze Fremdkörper, nicht nur die Bronchuswand sondern auch die Magen- oder Darmwand perforieren. Abführ- oder Brechmittel sind in diesem Fall absolut kontraindiziert. Wenn ein verschluckter Fremdkörper nicht nach drei Tagen vias naturales erscheint, sollte eine erneute radiologische Kontrolle erfolgen [14-16]. Die hier versäumte adäquate frühzeitige Diagnostik hatte für den Patienten nicht unerhebliche Konsequenzen, die dieser aufgrund seines hervorragenden Allgemeinzustandes sehr gut überstand.

\section{Schlussfolgerung}

Bei zahnärztlichen Behandlungen kann es trotz ausreichender Vorsichtsmaßnahmen zur Fremdkörperaspiration kommen. Um weiterreichende Komplikationen zu vermeiden, soltten unverzüglich die radiologische Lokalisationsdiagnostik und ggf. bronchoskopische Bergung veranlasst werden. Unerkannte Aspirate können auch nach mehreren symptomfreien Jahren zu chronischem Reizhusten führen und müssen differenzialdiagnostisch berücksichtigt werden. 


\section{Literatur}

${ }^{1}$ Baharloo F, Veyckemanns F, Francic C et al. Tracheobronchial foreign bodies: presentation and management in children and adults. Chest 1999; 115: 1357 - 1362

${ }^{2}$ Cameron SM, Whitlock WL, Tabor MS. Foreign body aspiration in dentistry: a review. J Am Dent Assoc 1996; 127: 1224-1229

${ }^{3}$ Czipull C, Reimer P. Intrapulmonaler metalldichter Fremdkörper. Radiologie 2003; 43: 672 - 676

${ }^{4}$ Eroglu A, Kurkcuoglu IC, Karaoglanoglu N et al. Tracheobronchial foreign bodies: a 10 years experience. Ulus Travma Derg 2003; 9: $262-266$

${ }^{5}$ Erren JP, Schipmann R. Right-sided recurrent penumonie of changing localization after aspiration of dental impression material with almost colplete casting of a segmental bronchus in a previously healthy woman. Pneumologie 1995; 49: 601-603

${ }^{6}$ Fishelberg G, Hook D. Patient safety during endodontic therapy using current technology. J Endod 2003; 29: 683-684

${ }^{7}$ Hedach F, Große-Sender S. Verschlucken und Aspiration von Fremdkörpern während der zahnärztlichen Behandlung. Int Poster J Dent Oral Med 2002; 4: 118
${ }^{8}$ Hodges ED, Durham TM, Stanley RT. Management of aspiration and Swallowing incidents: a review of the literature and report of case. ASDC J Dent Child 1992; 59: 413-419

${ }^{9}$ Hupp JR, Peterson LJ. Aspiration pneumonitis: etiology, therapy, and prevention. J Oral Surg 1981; 39: 430-435

${ }^{10}$ Kavanagh PV, Mason AC, Muller NL. Thoracic foreign bodies in adults. Clin Radiol 1999; 56: $353-360$

${ }^{11}$ Pritt B, Harman M, Schwart M et al. A tale of three aspirations: foreign bodies in the airway. J Clin Pathol. 2003; 56: $791-801$

${ }^{12}$ Seals ML, Andry JM, Kellar PN. Pulmonary aspiration of a metal casting: report of case. J Am Dent Assoc 1988; 117: 587-588

${ }^{13}$ Sopena B, Garcia-Caballero L, Diz P et al. Unsuspected foreign body aspiration. Quintessence Int 2003; 34: 779-781

${ }^{14}$ Swanson KL, Edell ES. Tracheobronchial foreign bodies. Chest Surg Clin N Am 2001; 11: 861 - 872

${ }^{15}$ Wunsch R, Wunsch C, Darge K. Foreign body aspiration. Radiologe 1999; 39: $467-471$

${ }^{16}$ Zitzmann NZ, Fried R, Elsasser S et al. The aspiration and swallowing of foreign bodies. The management of the aspiration or swallowing of foreign bodies during dental treatment. Schweiz Monatsschr Zahnmed 2000; 110: 619-632 